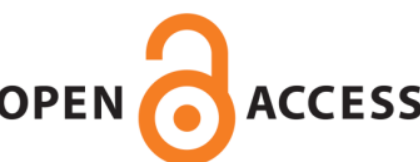

OPEN ACCESS

UWS Academic Portal

\title{
Complementary therapy for advanced dementia palliation in nursing homes
}

Mitchell, Bryan; Jackson, Graham A.; Sharp, Barbara; Tolson, Debbie

Published in:

Journal of Integrated Care

DOI:

10.1108/JICA-02-2020-0009

E-pub ahead of print: 16/06/2020

Document Version

Peer reviewed version

Link to publication on the UWS Academic Portal

Citation for published version (APA):

Mitchell, B., Jackson, G. A., Sharp, B., \& Tolson, D. (2020). Complementary therapy for advanced dementia palliation in nursing homes. Journal of Integrated Care, 28(4), 419-432. https://doi.org/10.1108/JICA-02-20200009

\section{General rights}

Copyright and moral rights for the publications made accessible in the UWS Academic Portal are retained by the authors and/or other copyright owners and it is a condition of accessing publications that users recognise and abide by the legal requirements associated with these rights. 


\section{$\underline{\text { Title }}$}

Complementary Therapy for Advanced Dementia Palliation in Nursing Homes

\section{Abstract}

- Purpose

This paper reports on an action research study that aimed to collaboratively develop a complementary therapy care intervention to augment palliative care choices available to nursing home residents with advanced dementia.

\section{- Design/Methodology/Approach}

An action research design was adopted that consisted of a series of action cycles involving collaborative exploration, problem solving planning, development and evidence gathering. A combination of mixed methods were used when gaining data at the different stages including face to face delivered questionnaires, observational notes, focus groups, and the objective measure of the Neuropsychiatric Inventory adapted for Nursing Homes (NPI-NH).

\section{- Findings}

Care home staff and relatives considered the use of Complementary Therapy to be a helpful intervention promoting that it can reduce a sense of loneliness and provide companionship for residents experiencing distress. Analysis of NPI-NH scores showed a reduction in presenting neuropsychiatric behaviours associated with stress and distress.

\section{- Research limitations/implications}

Differing levels of participant group engagement may affect this study's findings as it was noted that care home staff provided a fuller contribution to the project in comparison to relatives.

\section{- Practical implications}

Implementation guidance is needed when implementing complementary therapy within nursing home practice to promote consistency and successful integration of an intervention that is not provided as routine care.

\section{- Originality/value}

The findings of this study are encouraging and demonstrate the acceptability of complementary therapies to residents with advanced dementia, where positive impacts on otherwise difficult to address dementia symptoms related to stress and distress are highlighted.

\section{INTRODUCTION}

Dementia is an international public health priority, which places high demands on long- term care services in terms of both demand and the complexity of care. The policy imperative is to provide integrated health and social care that is personcentred and evidence informed, mindful of the relationships within care and caring. 
For people with advanced dementia integrated long-term care, would ideally include access to specialist palliative care (Murphy et al 2016; Tolson et al., 2017). Although there is increasing awareness of the need for advanced dementia palliation, inequalities exist when compared to conditions such as diabetes and cancer (Keohane and Petrie, 2019, Alzheimer Scotland 2019). An opportunity exists within care home practice to address some of this inequity by providing nonpharmacological interventions with the potential to provide comfort and alleviate advanced dementia related stress and distress (Palmer et al., 2018; Van der Steen, 2014). This is important as most care home residents with advanced dementia will experience dementia related stress and distress. Current guidance is to avoid inappropriate prescribing of antipsychotic medications when managing neuropsychiatric symptoms such as agitation and depression (Mulders et al 2016; Moyle et al., 2017).

Complementary Therapies such as the three listed below, have potential within dementia palliation as they can promote comfort and a sense of calm and could be administered within the care home environment:

- Body Massage including variations such as simple hand and arm, back, or lower leg and foot massage as informed by Beckman and Quesne (2005)

- Aromatherapy in the form of inhalations, compresses and as an accompaniment to the described forms of body massage influenced by Rhind (2012)

- Reflexology standard sessions informed by Crane (2012)

Complementary therapies of this nature have long been used in the palliative care setting (Macmillan Cancer Support, 2011) and as discussed by Tolson and Mitchell (2019), can be viewed as both a caring sensory intervention and a form of meaningful activity for people with advanced dementia.

The evidence for the use of complementary therapy for individuals with dementia is mainly anecdotal with a small number of published research studies (Wiles and Broker, 2019). Systematic reviews of the use of complementary therapy with dementia demonstrate variability in efficacy (Wu et al., 2017). Mitchell (2018) highlights that the existing research literature focusses on either pain relief or the specific neuropsychiatric symptom of agitation. The common data collection measure used is the Cohen Mansfield Agitation Inventory (CMAI) (Cohen-Mansfield, 1991) (Margenfeld et al., 2019) that relies on observable characteristics of a behaviour that cause distress for a caregiver, where other subtle neuropsychiatric behaviours that cause the individual with dementia distress can be difficult to detect and may be overlooked. Agitation is only one neuropsychiatric symptom among an array of others including depression, aggression, apathy, delusions, and hallucinations (Hu et al., 2015). 
This paper reports an action research study that aimed to collaboratively develop a complementary therapy care intervention to augment palliative care choices available to nursing home residents with advanced dementia (Mitchell 2018).

\section{METHODS}

\section{Design}

Action Research has been described as a strategy that engages researchers and participants within a community and co-generative process (Greenwood, 2007). Participation, partnership and collaboration are central tenets (Reason and Bradbury, 2013, 2001). This reported action research study adopted the mutual collaborative approach (Holter and Schwartz-Barcott, 1993) whereby the researcher and the participants worked collaboratively to explore and solve practice based challenges and issues which emerged as they sought to understand the potential of complementary therapies and how to use them within the care of residents with advanced dementia.

Once they had determined both the potential acceptability of selected complementary therapies and had prepared a person-centred administration protocol, a small number of individual resident case studies were prepared and influenced by the work of Yin (2014). The findings of these case studies with reference to impact on neuropsychiatric behaviours are the focus of this paper, which is contextualised within the fuller mixed method action research project.

\section{Study Aim}

The aim of this study was to;

'Collaboratively introduce and explore the contribution of complementary therapy within the care of nursing home residents experiencing later stages of dementia'.

As is typical of action research, the study was undertaken in a series of action cycles involving collaborative exploration, problem solving planning, development and evidence gathering. Working collaboratively with residents, visiting family and friends (VFF) and care home staff (CHS), the acceptability of different complementary therapy interventions was explored, alongside development for an administration protocol to tailor selected interventions to the preferences of individual residents. The emergent intervention protocol was then tested using a mixed methods approach including qualitative and quantitative methods within resident centred case studies (Mitchell 2018).

Two action cycles were conducted within two comparable independent sector nursing homes (A \& B) from different organisations. Nursing Home A was recruited based on existing professional relationships with the Nursing Home manager, where a keen interest to be involved in research was expressed. Nursing Home B was 
recruited based on similarities to the first Nursing Home where there were no existing relationships. The nursing homes were of similar size in terms of resident numbers, where each independent unit within either home resided 20 residents.

\section{Participants Eligibility}

To be an eligible member of CHS, individuals had to be permanent employees in a caring role (full or part time) of the care organisations. To be considered as a member of VFF, family or friends were required to have regular contact with the residing residents defined as a minimum of once per fortnight. Resident eligibility required a diagnosis of dementia and for the condition to be advanced. This was in addition to a minimum residency period of one month. Both participating homes believed that a period of one month was 'usually' enough time for a new resident to settle.

\section{Ethical Considerations}

Ethical approval was secured from [INSERT ETHICS COMMITTEE NAME] committee and [INSERT ETHICS COMMITTEE NAME]. Management permissions were also obtained from the providers and respective nursing home managers when accessing both sites.

For CHS and VFF, a standard consent process was adopted whereby participants were provided with information before being asked to provide written consent. However, when accessing and gaining consent on behalf of the residents involved in this research study, there was a crucial point to address, because all of the participants lacked the capacity to make the decision about whether or not to participate in research. Further details on the ethical considerations within this project are discussed at length elsewhere because as discussed in relation to the Adults with Incapacity (Scotland) Act 2000, incapacity is not an 'all or nothing' concept where one should not assume that because someone lacks capacity that they are now no longer able to make any decisions by themselves. In brief, a twostep process was followed where written consent was obtained from the welfare guardian or nearest relative for the purposes of research, where assent was further gained directly from the resident via verbal (if possible) or non-verbal cues.

\section{CYCLE 1}

Cycle 1 was undertaken in Nursing Home A and was considered as the preparatory cycle that was concerned with laying foundations and building a preliminary evidence base. This included exploring the views of CHS and VFF through face-to-face structured interviews to determine perceived acceptability and possible uses of three complementary therapies namely, Body Massage, Aromatherapy and Reflexology. Doing this provided a foundation and insight on how to move forward in a way that was inclusive of the multiple perspectives within a care home community. 
Five residents were recruited to test the intervention using person centred case studies. These case studies were based on two theoretical propositions:

- It is possible to provide person-centred complementary therapy interventions tailored to individual preferences within nursing home practice.

- Individualised complementary therapy has the potential to make a positive contribution to the care of residents with later stage dementia.

A selection of complementary therapies was introduced within the residents care plans providing a 30-60 minute long intervention, 2-3 times per week over 14 weeks. Residents were actively encouraged to select their intervention (if possible). Complementary therapy consultation records detailing the intervention session and any modifications to the individualised protocol in the moment of care, were recorded. In the first 30 minutes immediately following the intervention, staff were asked to note their own observations of resident behaviour adding to the consultation records. Relatives were invited to provide written feedback on a weekly basis reflecting on their thoughts about the impact, if at all, of the complementary therapy interventions sessions that their relative was receiving.

In addition to the therapist, staff and family views, the objective measurement tool of the Neuropsychiatric Inventory adapted for Nursing Homes (NPI-NH) was completed on a fortnightly basis by a trained nurse within the study site. The NPI-NH provides a brief assessment of neuropsychiatric symptomatology in clinical practice settings where observed changes in the NPI-NH scores were indicative of intervention impact at an individual resident level. The version used in the study has been modified for its specific use in extended care facilities that care for residents with dementia (Wood et al., 2002).

At the end of Cycle 1, the experience and the practicalities of introducing complementary therapy for individual residents was explored with $\mathrm{CHS}$ during a recorded focus group, and by individual telephone interviews with VFF.

\section{CYCLE 2}

Cycle 2, conducted in Nursing Home B, built upon the findings and lessons learned from the first action cycle. This new complementary therapy naïve nursing home community allowed us to further explore implementation lessons and to repeat the case studies to gain a better understanding of the impact on individual residents.

As action research is by nature emergent and negotiated, we began Cycle 2 by sharing findings from the first study home via a focus group with CHS. This focus group additionally had the purpose of co-constructing a set of implementation principles to prepare for the case studies. A brief questionnaire was prepared to capture views of staff who had been unable to participate in the focus group. As relationships between the researcher and care home community were developed the 
VFF and CHS requested taster sessions for themselves so that they could better understand how it felt to receive the different therapies. They felt this experience would better equip them to contribute to choices about which therapies the residents may benefit from and how they might be tailored towards an individual's needs should that person not be able to verbalise or make known their own preferences. Following this preparatory work, and with minor refinement to the administration protocol to capture staff and family views about resident potential intervention preferences, residents were recruited to participate in case studies using the previously described case study methods.

\section{RESULTS}

\section{Cycle 1 Findings}

When exploring CHS and VFF perceptions on the use of complementary therapy in nursing homes, $13 \mathrm{CHS}$ and $8 \mathrm{VFF}$ completed face to face structured interviews. These highlighted that both groups perceived the use of the three complementary therapy interventions to be acceptable in care homes for individuals experiencing later stages of dementia. This was under the proviso that when considering the implementation of complementary therapy that the interventions can be adapted to an individual's needs and circumstances, and reflect individual residents' wants and wishes, whilst at all times respecting their dignity.

Mindful of these perceptions, five individual case studies were developed. Residents who met the eligibility criteria as outlined in the participant eligibility section were recruited with the involvement and support of CHS, VFF following an ethically approved process.

Table 1 provides an overview of the included resident characteristics and interventions that were received within Nursing Home A.

\section{IINSERT TABLE 1: CYCLE 1 RESIDENT CHARACTERISTICS AND INTERVENTION OVERVIEW]}

The individual and group NPI-NH scores that were recorded in Nursing Home A during the case study period can be seen in Figure 1. Group scores were calculated on the mean average of all included residents. 
The $1^{\text {st }}, 2^{\text {nd }}$ and $3^{\text {rd }} \mathrm{NPI}-\mathrm{NH}$ 's recorded display the residents' symptoms over a 6week period before any complementary therapy intervention was introduced into their weekly care plan. On completion of the $3^{\text {rd }} \mathrm{NPI}-\mathrm{NH}$, Interventions were then introduced into said plans and given 4 weeks to become embedded. The NPI-NH was then completed again every 2 weeks for another 6 weeks, accounting for the $4^{\text {th }}$, $5^{\text {th }}$ and $6^{\text {th }} \mathrm{NPI}-\mathrm{NH}$.

In relation to the grouped resident NPI-NH results, there is a clear distinction in the NPI-NH scores, where residents' scores have reduced, demonstrating a positive sign. However, towards the end of the complementary therapy interventions the scores begin to rise. This gives the indication that potentially the complementary therapy interventions have a time frame where when the residents' become familiar with the therapy, the effects begin to reduce. Comparing the first NPI-NH average with the last, there is still a large difference in the presence of behaviour as recorded via the scores. All residents who experienced a change in behaviour, experienced a positive one, whereby scores were reduced.

Over the duration of the intervention phase, 31 comments were made on the 57 interventions that were provided to residents by $\mathrm{CHS}$. The majority of the comments point to the intervention having a positive impact, where several words continuously reappear such as 'relaxed' 'settled', and 'content'. However, on very few occasions, it was noted that the intervention did not make a difference. Only two comments were offered from VFF. These member of VFF believed the residents' enjoyed receiving the intervention. However, it was also noted from both comments that VFF did not see any noticeable differences in their relative's overall behaviour.

When reflecting on the experiences and practicalities of the case study process, 5 CHS participated in a focus group and 3 VFF via individual telephone interviews. Both groups reflected that the one to one time that residents were receiving during these interventions were a major benefit to the process, with staff additionally commenting that some of their work pressures were relieved due to the additional support being provided to residents. A criticism included that VFF felt the communicative dialogue between staff and themselves could have been better concerning the complementary therapy interventions providing a possible reason for poor VFF engagement.

\section{Cycle 2 Findings}

In Nursing Home B, $10 \mathrm{CHS}$ collaboratively worked with the summary script detailing the findings from Nursing Home A and co-constructed principles of implementation. This equated to $5 \mathrm{CHS}$ during the initial focus group and an additional $5 \mathrm{CHS}$ via the additional questionnaire. The agreed principles were that the complementary therapist should. 
- Take a holistic view by acknowledging the whole person rather than just the symptoms presented.

- Be flexible in their approach and respond to an individual's needs.

- Work collaboratively with CHS, VFF, and residents, to promote successful implementation.

- Be transparent and provide feedback on the complementary therapy interventions to both CHS and VFF.

- Be appropriately qualified when administering and undertaking the complementary therapy interventions.

These principles of implementation would be the foundation for any nursing home to consider when introducing complementary therapy into the environment.

Mindful of the principles of implementation, ten residents were recruited to participate in further individual case studies. Table 2 provides an overview of the included resident characteristics and interventions that were received within Nursing Home B.

\section{IINSERT TABLE 2: CYCLE 2 RESIDENT CHARACTERISTICS AND INTERVENTION OVERVIEW]}

The individual and group NPI-NH scores that were recorded in Nursing Home B during the case study period can be seen in Figure 2. Group scores were calculated on the mean average of all included residents.

\section{[INSERT FIGURE 2: NURSING HOME B: NPI-NH RESULTS]}

The $1^{\text {st }} \mathrm{NPI}-\mathrm{NH}$ acted as the baseline for resident's symptoms. On completion of the $1^{\text {st }}$, Interventions were then introduced into the resident's weekly care plans for approximately 10 weeks. Resident K's data was excluded from the result because the resident sadly died during the study period. Resident L's data has also been excluded from the grouped resident NPI-NH results because of unusually high NPI$\mathrm{NH}$ scores. Nursing staff made the researcher aware that Resident $L$ was potentially experiencing an episode of delirium throughout the process due to a Urinary Tract Infection. Residents $\mathrm{G}, \mathrm{L}, \mathrm{O}$ and $\mathrm{P}$ had a delay to starting the NPI-NH recording process where they were approximately 2 weeks behind, resulting in only $4 \mathrm{NPI}-\mathrm{NH}$ results being recorded. The grouped resident NPI-NH figure therefore only displays four averaged NPI-NH points for all included residents as opposed to five.

There is a clear indication that the complementary therapy intervention has impacted on the neuropsychiatric behaviours presented by residents in a positive way. From the first NPI-NH completed in comparison to the last, only one resident (Resident $\mathrm{L}$ ) 
experienced an increase in their presenting behaviours. As highlighted, this unusually high score could have been caused by other health issues.

Over the duration of the intervention phase, 21 comments were made on the 118 interventions that were provided to residents by $\mathrm{CHS}$ at fortnightly intervals as opposed to the previous cycles 30-minute post observation. From the responses given, it becomes clear that residents seemed to enjoy and welcome having the intervention. Similar words from the previous cycle continuously reappear such as 'relaxed' 'comforting', and 'attention'. Only one comment was made from VFF which described the intervention as mentioning also that the family believed that the intervention had increased the resident's overall quality of life.

\section{DISCUSSION}

The study has found that it was possible to introduce complementary therapy into the care of nursing home residents with advanced dementia in ways that were reflective of individual preference and choice. For participating residents, this innovation in care appeared to be welcome and associated with observable benefits as demonstrated through a reduction in neuropsychiatric symptoms reflected in NPI-NH scores and perceived benefits through a sense of companionship that offset feelings of loneliness. Given that these benefits were evident from interventions delivered on average twice weekly over 30-60 minutes, these findings are encouraging and indicative of the potential of complementary therapy within advanced dementia care palliation. These findings align to the policy rhetoric worldwide where national and international organisations are calling for the development of evidence-based nonpharmacologic interventions for people with dementia, with the aims of supporting those experiencing neuropsychiatric symptoms, stimulating cognitive function, quality of life (World Health Organisation, 2015).

The NPI-NH results taken in isolation should be interpreted with caution because it was never fully considered in this study as a standalone outcome measure. The NPI$\mathrm{NH}$ itself requires commitment to a longer period as well as a larger sample to have any significant impact on the results. However, nursing staff who completed the NPI$\mathrm{NH}$ were trained and provided with information on how to use the measurement tool in practice. In addition to this, all NPI-NH scores for individual residents were provided from one member of nursing staff (one rater per resident). This promoted the inter-reliability of scores because of consistency in the information provided to staff across homes, and consistency in scores being recorded for residents, removing differences of opinions from each member of nursing staff. As discussed in (Iverson et al., 2002), a point to be mindful of is that a beneficial clinically meaningful change in the Neuropsychiatric Inventory has been defined as plus or minus 9 points in an individual residents score. As some residents in this study did not have a score of above 9 at baseline suggests that their score has had no significance overall. 
However, the NPI-NH scores taken in tandem with other data sets in the individual resident case studies are seen as a useful objective and practical indicator to demonstrate the potential of complementary therapy impacting on neuropsychiatric behaviours related to dementia.

The objectivity of using the NPI-NH was considered superior to reliance on staff's subjective views about the impact of the intervention with individual residents. Therefore, it is reasonable to speculate that an improvement in NPI-NH scores is indicative of a satisfactory or good experience of care.

The NPI-NH was acceptable to use with the nursing staff in this environment as they were involved when selecting which objective measure to use within practice.

\section{Loneliness and Companionship}

When identifying and selecting residents to participate in the case studies within both nursing homes, both companionship and loneliness were considered key factors that influenced CHS and VFF decision-making. In total, nine out of the fifteen residents across both homes were recruited with the primary reason being related to loneliness and companionship. Varying reasons such a lack of family or friend visitation, or a need for more one to one time were noted. Other reasons included, four being identified as participants due to staff feeling they may help behavioural symptoms, one recruited due to likelihood of enjoyment from therapy, and one for supporting mobility issues. In this study it was not clear if this was a consequence of not understanding the intended therapeutic benefit of complementary therapy, or an insightful recognition of the link between a comforting experience, a sense of wellbeing, connecting and human contact that is central to complementary therapy. As discussed in the literature, feelings of loneliness may lead to stress because of the absence of social integration (Burke and Segrin, 2014). Loneliness itself has been shown to affect quality of sleep, reduce immune system effectiveness, and increase stress responses (Masi et al., 2011). It could therefore have been possible, that CHS and VFF may have additionally linked the need for residents to have social integration of this form in their care plans. These contributing factors were not originally considered because the literature that was reviewed in terms of nonpharmacological interventions moved towards reducing medications, stress, distress, pain and dementia-related behaviour (Wilfling et al., 2015; Payne et al., 2012; O'Brien et al., 2011). However, the alleviation of loneliness and isolation is a major priority that is likely to remain high on the policy agenda with an ageing population (Grant, 2016) and potentially should be considered when considering the use of these interventions.

From the surrounding literature on loneliness and social isolation, what we currently know is that it is a common issue among older people and those in palliative care (Perissinotto et al., 2019; Golden et al., 2009), is a growing concern to society 
(Holwerda et al., 2016) and is strongly associated with adverse health effects such as depression, physical impairment, quality of life, cognitive decline and increased mortality (Theeke, 2010). Gierveld et al (2016) further add that in the past decade, the several reviews of the effectiveness of loneliness interventions that have been published repeatedly arrive at the conclusion that few existing interventions successfully reduce loneliness and social isolation. Additionally, to date, no study has looked at complementary therapy in relation to loneliness and social isolation or providing comfort. There is a lack of deep understanding of the nature of loneliness and based on the findings and experience gained in this study as well as existing literature, it would suggest that this is particularly true for nursing home residents experiencing advanced dementia, and those in palliative care (Victor, 2012).

The data sets across both nursing homes had also highlighted that the predominant choice of intervention was variations of massage where there is a need for physical contact. This is possibly the residents' own way of expressing a need for human contact and connection. It is important to appreciate that just a decade ago, the role of touch in nursing was often only associated with tasks related to delivering physical care or clinical procedures (Dean et al., 2017). Touch has already been recognised as an integral part to communicating care, comfort and compassion and evidence is growing of its importance within the patient experience (Bensing et al., 2013; Deledda et al., 2013), however, this study highlights that although literature might recognise the importance of touch, in practice its use is variable as is awareness of its therapeutic potential.

\section{IMPLICATIONS}

\section{Research Limitations}

A limitation that may affect the findings from this study might be the differing levels of participant group engagement. This study was intended to be a collaborative project, achieving shared project decision making and planning with CHS and VFF from both participating nursing homes. This has not been the case because the $\mathrm{CHS}$ provided a fuller contribution to the project in comparison to that of the VFF based on anticipated levels of participation as set out by the researcher. When trying to engage VFF, many of whom are older themselves, it was perhaps unrealistic to anticipate that they would engage at the same level as staff. Additionally, as lack visitation from VFF was a selection criterion for some residents, a reduce contribution would therefore happen. However, if contribution from the participant groups was balanced, the results may have differed.

The intervention protocol has been developed, refined and tested under standard care home practice conditions as part of an emergent action research design. The encouraging findings suggest that complementary therapy has potential and is viable 
within dementia palliation as part of care home practice but further testing under trial conditions would be warranted.

\section{Implications for Practice}

When implementing Complementary Therapy within Nursing Homes for individuals with advanced dementia, this study has highlighted that certain considerations must be made. These are a need to develop implementation guidelines for nursing homes concerning eligibility and applicability of residents. This would support the integration of a therapeutic intervention that is not offered as routinely provided care and ensures consistency of practice across care.

Regardless of the circumstances in which complementary therapy is being used (pain, advanced dementia palliation), its provision needs to be considered, bearing in mind that it possesses contraindications and risks that could be harmful to residents. Working collaboratively with CHS throughout both nursing homes, and being informed by the findings from this study, a checklist for CHS has been developed with the aim of providing a criteria to determine if complementary therapy is suitable for the nursing home and the individual residents. The checklist is available on request from the authors.

\section{CONCLUSION}

The findings of this study are encouraging and demonstrate the acceptability of complementary therapies to participating residents with advanced dementia. Statistical findings have indicated through the use of the objective measure of the $\mathrm{NPI}-\mathrm{NH}$ that complementary therapy may play a role in supporting residents who are experiencing neuropsychiatric behaviours by alleviating and reducing their presenting neuropsychiatric symptoms. Although the results from the measure are positive, further research is needed under controlled circumstances to test the clinical efficacy of complementary therapy on neuropsychiatric behaviours. The key qualitative findings of this study highlighted the potential of complementary therapy being utilised and introduced into residents' weekly care plans as a therapeutic intervention to offset loneliness and provide companionship. Similarly, further research is recommended to more fully understand the potential benefits of the original caring intervention protocol developed through this study.

The findings produced remain promising in the pursuit for a broader range of nonpharmacological interventions with therapeutic intention for people with advanced dementia by highlighting potential uses of complementary therapy and demonstrating that they can be implemented into practice successfully.

\section{DECLARATION OF CONFLICTING INTERESTS}


The author(s) declared no potential conflicts of interest with respect to the research, authorship, and/or publication of this article

\section{REFERENCE LIST}

Beckmann, H., and Le Quesne, S. E. (2005) The Essential Guide to Holistic and Complementary Therapy. London. Thomson Learning.

Bensing, J., Rimondini, M., and Visser, A. (2013) What Patients Want. Patient Education and Counselling. Vol.90 (3), pp.287-290

Burke, T.J., \& Segrin, C. (2014). Bonded or stuck? Effects of personal and constraint commitment on loneliness and stress. Personality and Individual Differences, Vol.64, pp.101-106.

Cerejeira, J., Lagarto, L., and Mukaetova-Ladinska, E. (2012) Behavioural and psychological symptoms of dementia. Frontiers in Neurology. Vol.3 p.73.

Cohen-Mansfield, J. (1991) Instruction manual for the Cohen-Mansfield agitation inventory (CMAI). Research Institute of the Hebrew Home of Greater Washington.

Crane, B. (2012) Reflexology: The Definitive Practitioner's Manual: Recommended by the International Therapy Examination Council for Students and Practitioners. HarperCollins UK.

Dean, S., Lewis, J., and Ferguson, C. (2017) Is technology responsible for nurses losing touch? Journal of Clinical Nursing. Vol.26 (5-6), pp.583-585.

Deledda, G., Moretti, F., Rimondini, M., and Zimmermann, C. (2013) How patients want their doctor to communicate. A literature review on primary care patients' perspective. Patient Education and Counseling. Vol.90 (3), pp.297-306.

Golden, J., Conroy, R. M., Bruce, I., Denihan, A., Greene, E., Kirby, M., and Lawlor, B. A. (2009) Loneliness, social support networks, mood and wellbeing in communitydwelling elderly. International Journal of Geriatric Psychiatry. Vol.24 (7), pp.694-700.

Grant, C. (2016). Loneliness and social isolation in older people. [ONLINE] Available at:http://www.lgiu.org.uk/wp-content/uploads/2016/02/Loneliness-and-

socialisolation.pdf. [Last Accessed 1st February 2017]

Greenwood, D.J., (2007). Pragmatic action research. International Journal of Action Research, 3(1-2), pp.131-148.

Holwerda, T.J., van Tilburg, T.G., Deeg, D.J., Schutter, N., Van, R., Dekker, J., Stek, M.L., Beekman, A.T. and Schoevers, R.A., (2016). Impact of loneliness and depression on mortality: results from the Longitudinal Ageing Study Amsterdam. The British Journal of Psychiatry, 209 (2), pp.127-134.

Hu, R.F., Jiang, X.Y., Chen, J., Zeng, Z., Chen, X.Y., Li, Y., Huining, X. and Evans, D.J., (2015). Non-pharmacological interventions for sleep promotion in the intensive care unit. Cochrane Database of Systematic Reviews, (10). pp.1-101. 
Iverson, G.L., et al., (2002). Measuring change in psychiatric symptoms using the Neuropsychiatric Inventory: Nursing Home version. International Journal of Geriatric Psychiatry, 17 (5), pp.438-443.

Keohane, N., Pertie, K., (2019) Social Market Foundation: Creating a Dementia Fund. Alzheimer Society. pp.1-42.

Macmillan Cancer Support. (2011) Cancer and Complementary Therapies (seventh ed), London (2011).

Margenfeld, F., Klocke, C. and Joos, S., (2019). Manual massage for persons living with dementia: A systematic review and meta-analysis. International Journal of Nursing Studies. Vol 99. pp.132-142.

Masi, C. M., Chen, H.-Y., Hawkley, L. C., \& Cacioppo, J. T. (2011). A meta-analysis of interventions to reduce loneliness. Personality and Social Psychology Review, 15, pp.219-266.

Mitchell, B. (2018). 'The Contribution of Complementary Therapy within the Care of Nursing Home Residents Experiencing Later Stages of Dementia'. Doctoral Thesis. University of the West of Scotland. Lanarkshire.

Moyle, W., El Saifi, N., Draper, B., Jones, C., Beattie, E., Shum, D., Thalib, L., Mervin, C. and Dwyer, S., 2017. Pharmacotherapy of persons with dementia in longterm care in Australia: A descriptive audit of central nervous system medications. Current Drug Safety, 12 (2), pp.95-102.

Mulders, A.J., Fick, I.W., Bor, H., Verhey, F.R., Zuidema, S.U. and Koopmans, R.T., (2016). Prevalence and correlates of neuropsychiatric symptoms in nursing home patients with young-onset dementia: the BEYOnD study. Journal of the American Medical Directors Association, 17 (6), pp.495-500.

Murphy, E., Froggatt, K., Connolly, S., O'Shea, E., Sampson, E.L., Casey, D. and Devane, D., (2016). Palliative care interventions in advanced dementia. Cochrane database of systematic reviews, (12). pp.1-44.

O'Brien, J. T., and Burns, A. (2011) Clinical practice with anti-dementia drugs: a revised (second) consensus statement from the British Association for Psychopharmacology. Journal of Psychopharmacology. Vol.25 (8), pp.9971019.

Palmer, J.A., Parker, V.A., Berlowitz, D., Snow, A.L. and Hartmann, C.W., (2018).

Resident choice: a nursing home staff perspective on tensions and resolutions. Geriatric Nursing, 39(3), pp.271-278.

Payne, C., Wiffen, P.J. and Martin, S., (2012). Interventions for fatigue and weight loss in adults with advanced progressive illness. Cochrane Database of Systematic Reviews, (1). pp.1-39.

Reason, P. and Bradbury, H., (2001). Introduction: Inquiry and participation in search of a world worthy of human aspiration. Handbook of action research: Participative inquiry and practice. 
Reason, P. and Bradbury, H., (2013). Introduction: Inquiry and participation in search of a world worthy of human aspiration. Handbook of action research: Participative inquiry and practice.

Rhind, J. P. (2012) Essential Oils: A Handbook for Aromatherapy Practice. 2nd Ed. London: Sage.

The Scottish Parliament. (2000). Adults with Incapacity (Scotland) Act 2000. Queen's Printer for Scotland.

Theeke, L. A. (2010) Sociodemographic and health-related risks for loneliness and outcome differences by loneliness status in a sample of US older adults. Research in Gerontological Nursing. Vol.3(2), pp.113-125.

Tolson, D., Holmerova, I., Macrae, R., Waugh, A., Hvalič-Touzery, S., de Abreu, W., Crespo, M.L., Merta, A. and Hanson, E., (2017). Improving advanced dementia care: An interprofessional palliare learning framework. Journal of the American Medical Directors Association, 18(7), pp.561-563.

Van der Steen, J.T., Dekker, N.L., Gijsberts, M.J.H., Vermeulen, L.H. and Mahler, M.M., (2017). Palliative care for people with dementia in the terminal phase: a mixedmethods qualitative study to inform service development. BMC palliative care, 16(1), p.28.

Victor, C.R., (2012). Loneliness in care homes: a neglected area of research? Aging health, 8(6), pp.637-646.

Wiles, A., and Broker, D., (2019). 38 Complementary Therapies in Dementia Care. Perspectives on Complementary and Alternative Medicine: A Reader, p.7.

Wilfling, D., Junghans, A., Marshall, L., Eisemann, N., Meyer, G., Möhler, R., and Köpke, S. (2015) Non-pharmacological interventions for sleep disturbances in people with dementia. The Cochrane Library. pp.1-12.

Woods, D. L., and Dimond, M. (2002) The effect of therapeutic touch on agitated behavior and cortisol in persons with Alzheimer's disease. Biological Research for Nursing. Vol.4 (2), pp.104-114.

World Health Organization, (2015). First WHO ministerial conference on global action against dementia: meeting report, WHO Headquarters, Geneva, Switzerland, 16-17 March 2015.

Wu, J., Wang, Y. and Wang, Z., (2017). The effectiveness of massage and touch on behavioural and psychological symptoms of dementia: A quantitative systematic review and meta-analysis. Journal of advanced nursing, 73(10), pp.2283-2295.

Yin, R.K., (2014). Case study research: Design and methods (applied social research methods). Thousand Oaks, CA: Sage publications. 


\begin{tabular}{|c|c|c|c|c|c|c|c|c|}
\hline \multicolumn{9}{|c|}{ Cycle 1: Nursing Home A } \\
\hline \multicolumn{9}{|c|}{ Resident Characteristics } \\
\hline & Age & \multicolumn{2}{|l|}{$\begin{array}{l}\text { Length of } \\
\text { Admission }\end{array}$} & \multicolumn{2}{|c|}{$\begin{array}{l}\text { Diagnosi } \\
s\end{array}$} & \multicolumn{2}{|c|}{ Type (if known) } & Completed \\
\hline Resident A & 91 & \multicolumn{2}{|l|}{2.4 years } & \multicolumn{2}{|c|}{ Yes } & \multicolumn{2}{|c|}{ Alzheimer's } & Yes \\
\hline Resident B & 93 & \multicolumn{2}{|l|}{2.4 years } & \multicolumn{2}{|c|}{ Yes } & \multicolumn{2}{|c|}{ Unknown } & Yes \\
\hline Resident C & 91 & \multicolumn{2}{|l|}{6.4 years } & \multicolumn{2}{|c|}{ Yes } & \multicolumn{2}{|c|}{ Unknown } & Yes \\
\hline Resident D & 85 & \multicolumn{2}{|l|}{4.9 years } & \multicolumn{2}{|c|}{ Yes } & \multicolumn{2}{|c|}{ Unknown } & Yes \\
\hline Resident E & 82 & \multicolumn{2}{|l|}{4.10 years } & \multicolumn{2}{|c|}{ Yes } & \multicolumn{2}{|c|}{ Alzheimer's } & Yes \\
\hline \multicolumn{9}{|c|}{ Intervention Overview } \\
\hline & & & \multicolumn{6}{|c|}{ Resident } \\
\hline & & & $A$ & $B$ & $C$ & $D$ & $E$ & Total \\
\hline \multicolumn{3}{|c|}{ Variations of Massage } & 4 & 11 & 4 & 15 & 9 & 43 \\
\hline \multicolumn{3}{|l|}{ Reflexology } & 8 & 0 & 6 & 0 & 0 & 14 \\
\hline \multicolumn{3}{|c|}{ Aromatherapy (Solo) } & 0 & 0 & 0 & 0 & 0 & 0 \\
\hline \multicolumn{3}{|c|}{ Aromatherapy (Addition) } & $\checkmark$ & $\checkmark$ & $\checkmark$ & $\checkmark$ & $x$ & \\
\hline \multicolumn{3}{|c|}{ Total Received } & 12 & 11 & 10 & 15 & 9 & 57 \\
\hline
\end{tabular}



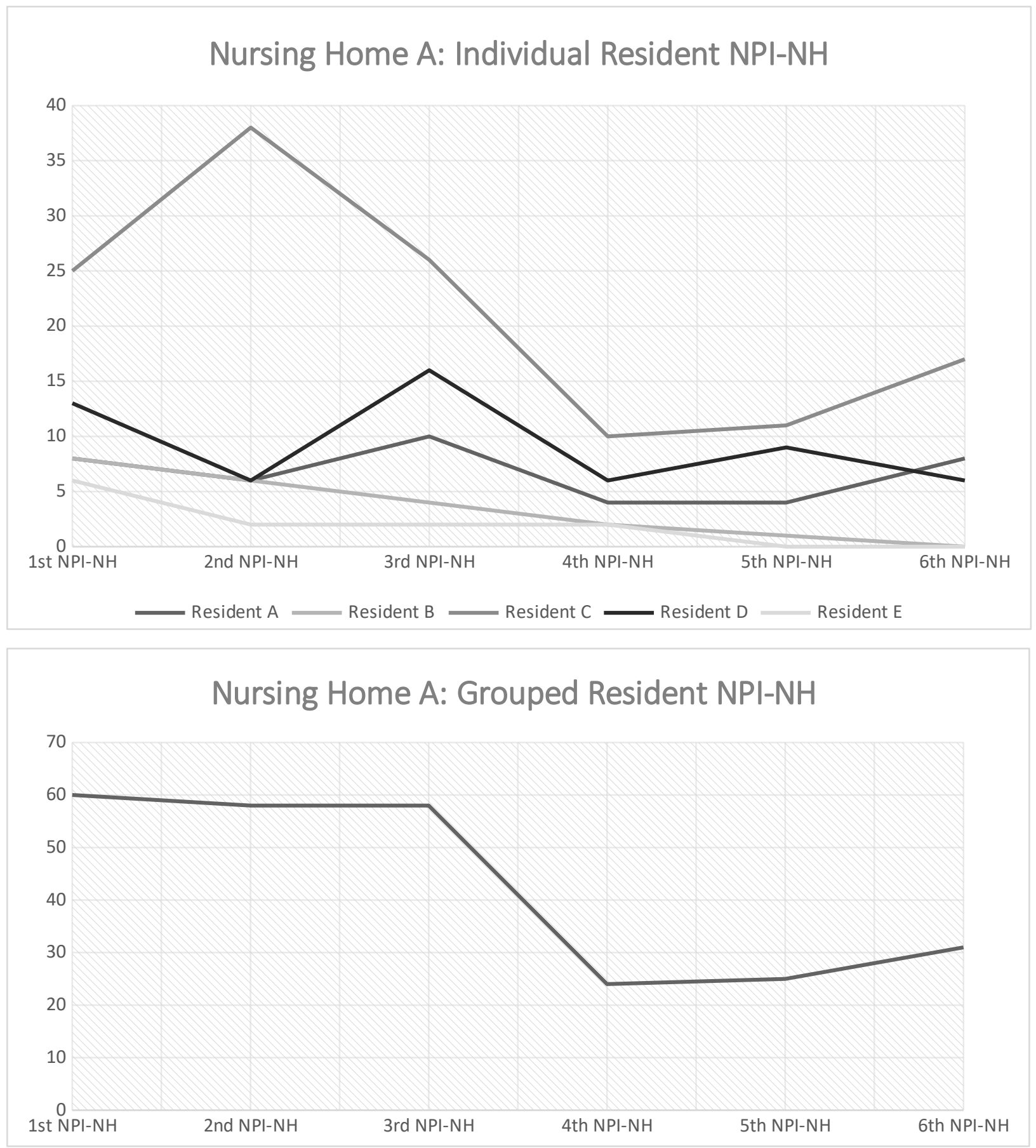


\begin{tabular}{|c|c|c|c|c|c|c|c|c|c|c|c|c|}
\hline \multicolumn{13}{|c|}{ Cycle 2: Nursing Home B } \\
\hline \multicolumn{13}{|c|}{ Resident Characteristics } \\
\hline & Age & \multicolumn{4}{|c|}{$\begin{array}{l}\text { Length of } \\
\text { Admission }\end{array}$} & \multicolumn{2}{|c|}{$\begin{array}{l}\text { Diagnosi } \\
s\end{array}$} & \multicolumn{4}{|c|}{ Type (if known) } & Completed \\
\hline Resident G & 83 & \multicolumn{4}{|c|}{3 years } & \multicolumn{2}{|c|}{ Yes } & \multicolumn{4}{|c|}{ Alzheimer's } & Yes \\
\hline Resident H & 71 & \multicolumn{4}{|c|}{11 months } & \multicolumn{2}{|c|}{ Yes } & \multicolumn{4}{|c|}{ Lewy Body } & Yes \\
\hline Resident I & 87 & \multicolumn{4}{|c|}{3 years } & \multicolumn{2}{|c|}{ Yes } & \multicolumn{4}{|c|}{ Unknown } & Yes \\
\hline Resident J & 81 & \multicolumn{4}{|c|}{2 years } & \multicolumn{2}{|c|}{ Yes } & \multicolumn{4}{|c|}{ Unknown } & Yes \\
\hline Resident K & 84 & \multicolumn{4}{|c|}{2 years } & \multicolumn{2}{|c|}{ Yes } & \multicolumn{4}{|c|}{ Alzheimer's } & No \\
\hline Resident L & 88 & \multicolumn{4}{|c|}{2 years } & \multicolumn{2}{|c|}{ Yes } & \multicolumn{4}{|c|}{ Alzheimer's } & Yes \\
\hline Resident M & 81 & \multicolumn{4}{|c|}{5 years } & \multicolumn{2}{|c|}{ Yes } & \multicolumn{4}{|c|}{ Unknown } & Yes \\
\hline Resident N & 72 & \multicolumn{4}{|c|}{8 months } & \multicolumn{2}{|c|}{ Yes } & \multicolumn{4}{|c|}{ Alzheimer's } & Yes \\
\hline Resident $\mathbf{O}$ & 86 & \multicolumn{4}{|c|}{7 months } & \multicolumn{2}{|c|}{ Yes } & \multicolumn{4}{|c|}{ Unknown } & Yes \\
\hline Resident $\mathbf{P}$ & 80 & $5 y$ & ears & & & $Y e$ & & & Kno & & & Yes \\
\hline Intervention & Over & iew & & & & & & & & & & \\
\hline & & Res & dent & & & & & & & & & \\
\hline & & $G$ & $\mathrm{H}$ & I & $\mathrm{J}$ & $\mathrm{K}$ & L & $M$ & $\mathrm{~N}$ & 0 & $P$ & Total \\
\hline $\begin{array}{l}\text { Variations of } \\
\text { Massage }\end{array}$ & & 13 & 0 & 8 & 10 & 4 & 0 & 0 & 24 & 8 & 9 & 76 \\
\hline Reflexology & & 1 & 20 & 0 & 4 & 0 & 7 & 10 & 0 & 0 & 0 & 42 \\
\hline $\begin{array}{l}\text { Aromatherapy } \\
\text { (Solo) }\end{array}$ & & 0 & 0 & 0 & 0 & 0 & 0 & 0 & 0 & 0 & 0 & 0 \\
\hline $\begin{array}{l}\text { Aromatherapy } \\
\text { (Addition) }\end{array}$ & & $\checkmark$ & $\checkmark$ & $\checkmark$ & $\checkmark$ & $\checkmark$ & $\checkmark$ & $\checkmark$ & $\checkmark$ & $\checkmark$ & $\checkmark$ & \\
\hline Total Receiveo & & 14 & 20 & 8 & 14 & 4 & 7 & 10 & 24 & 8 & 9 & 118 \\
\hline
\end{tabular}




\section{Nursing Home B: Individual Resident NPI-NH}

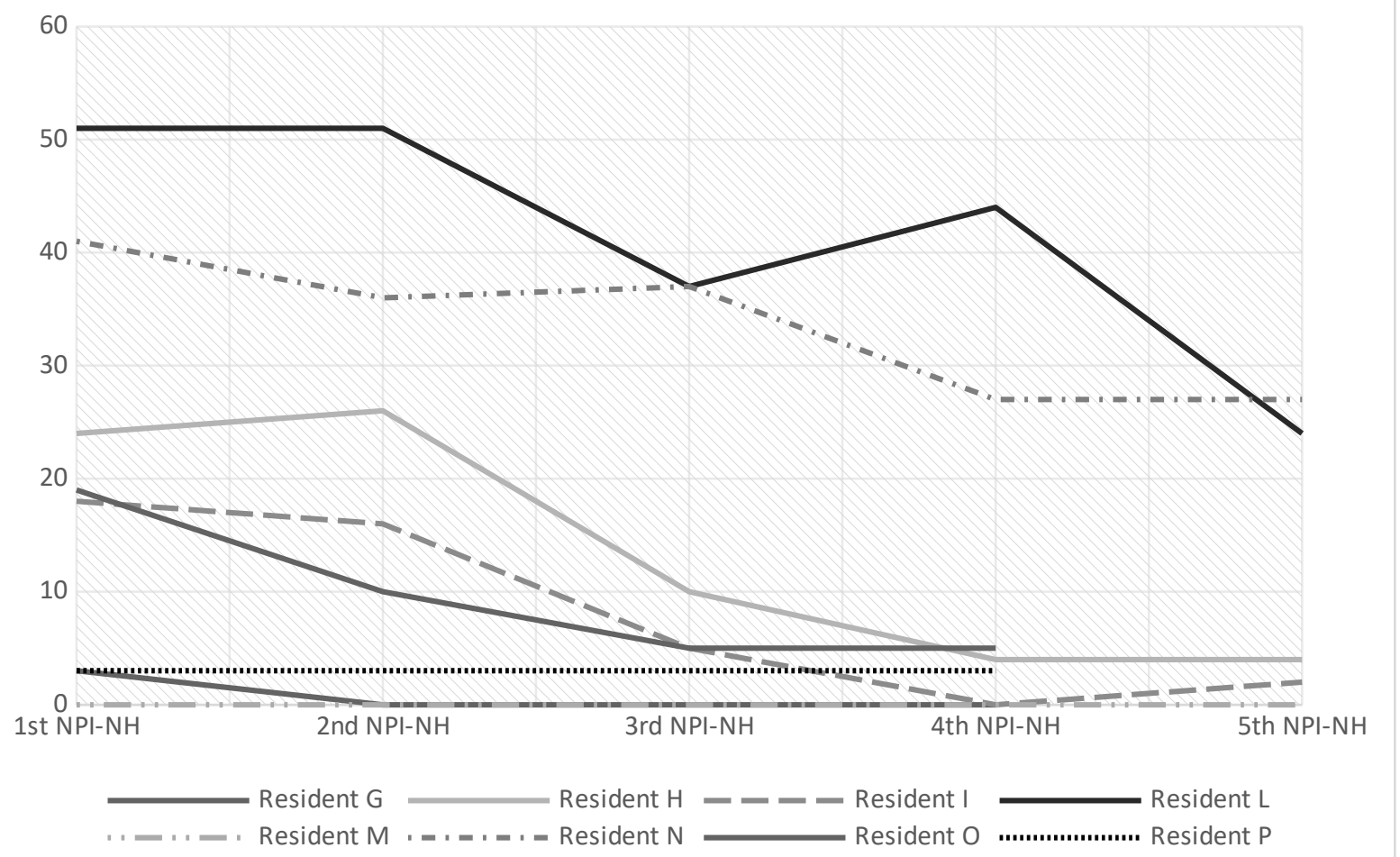

\section{Nursing Home B: Grouped Resident NPI-NH}

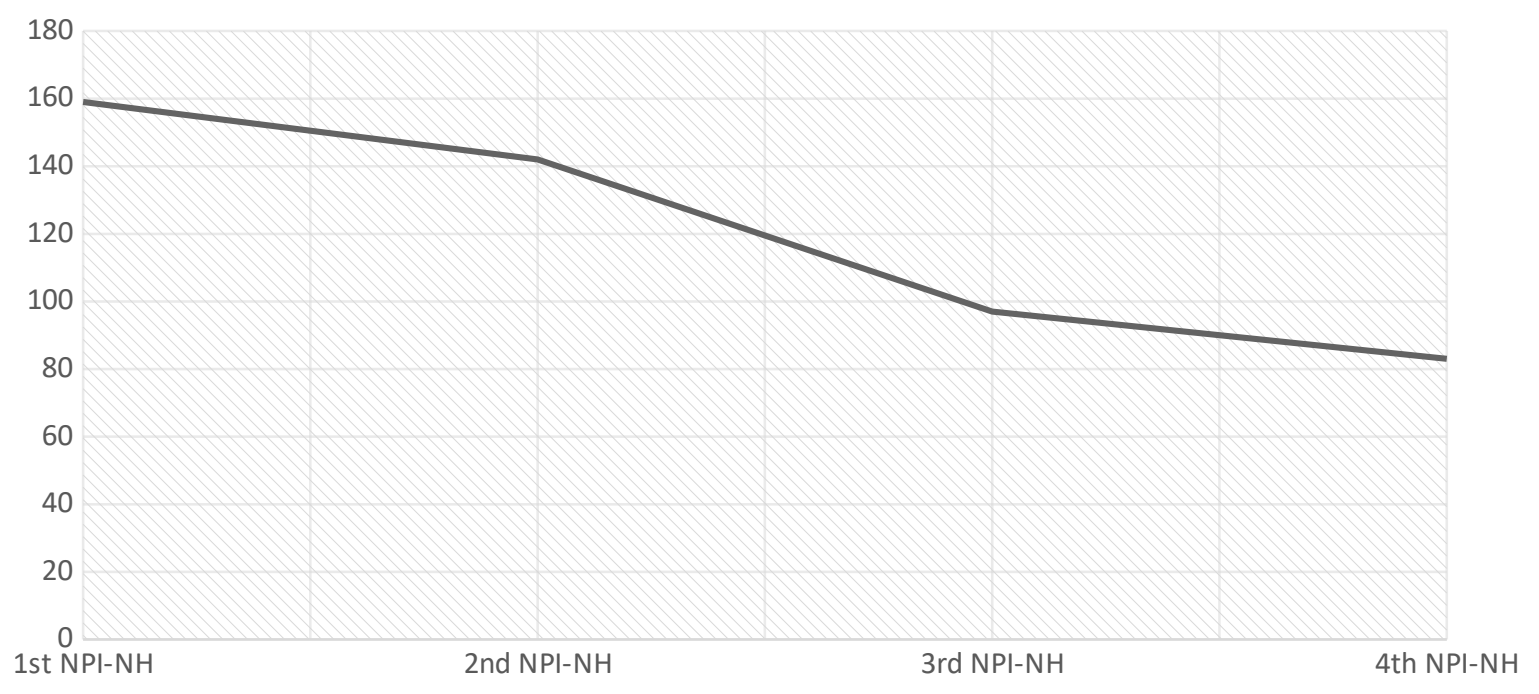

